\title{
Atangana-Baleanu and Caputo-Fabrizio Analysis of Fractional Derivatives on MHD Flow past a Moving Vertical Plate with Variable Viscosity and Thermal Conductivity in a Porous Medium
}

\author{
Dipen Saikia \\ Department of Basic and Applied Science, \\ National Institute of Technology Arunachal Pradesh, Yupia, Arunachal Pradesh, India. \\ E-mail: deepsand2009@gmail.com \\ Utpal Kumar Saha \\ Department of Basic and Applied Science, \\ National Institute of Technology Arunachal Pradesh, Yupia, Arunachal Pradesh, India. \\ Corresponding author: utpal@ nitap.ac.in \\ Gopal Chandra Hazarika \\ Department of Mathematics, \\ Dibrugarh University, Dibrugarh, Assam, India. \\ E-mail: gchazarika@dibru.ac.in
}

(Received April 6, 2020; Accepted September 8, 2020)

\begin{abstract}
In this paper, a numerical investigation is presented for non-integer order derivatives with Atangana-Baleanu (AB) and Caputo-Fabrizio (CF) fractional derivatives for the variable viscosity and thermal conductivity over a moving vertical plate in a porous medium two dimensional free convection unsteady MHD flow. The effects of radiation have also been considered. The governing partial differential equations along with the boundary conditions are changed to ordinary form by similarity transformations. Hence physical parameters show up in the equations and interpretations on these parameters can be achieved suitably.By using ordinary finite difference scheme the equations are discritized and developed in fractional form. These discritized equations are numerically solved by the approach based on Gauss-seidel iteration scheme. Some numerical strategies are used to find the values of $\mathrm{AB}$ and $\mathrm{CF}$ approaches on time by developing programming code in MATLAB. The effects of all the physical parameters involved in the problem on velocity, temperature and concentration distribution are compared graphically as well as in tabular form. The effects of each parameter are found to be prominent. We have observed a significant variation of values under different parameters using $\mathrm{AB}$ and $\mathrm{CF}$ approaches on velocity, temperature and concentration distribution with respect to time.
\end{abstract}

Keywords- AB and CF derivatives, Viscosity, Thermal conductivity, Porous medium, Radiation.

\section{Introduction}

The boundary layer and heat transfer flow of a viscous fluid over a moving vertical plate in a porous medium have been investigated in a number of technological approaches such as warm rolling, metallic extrusion, petroleum industries, polymer extrusion, wires drawing and metallic spinning. Natural convection flows driven by temperature differences are of great interest in a number of industrial applications. Bejan and Khair (1985) studied free convection heat and mass transfer in a porous medium. In recent there has been a growing interest in studing the combined application of MHD flow and porous media. Aldoss et al. (1995) investigated combined free and forced convection flow from vertical plate in aporous medium in the presence of magnetic field. Hossain and Munir (2000) analysed a two dimensional mixed convection flow of a viscous incompressible fluid of temperature dependent viscosity past a vertical plate. 
International Journal of Mathematical, Engineering and Management Sciences

Vol. 6, No. 2, 493-509, 2021

https://doi.org/10.33889/IJMEMS.2021.6.2.030

Javaherdeh et al. (2015) studied the natural convection heat and mass transfer in MHD fluid flow past a moving vertical plate with variable surface temperature and concentration in a porous medium. However, the impact of variable viscosity and thermal conductivity of a MHD free convection flow past a vertical plate embedded in porous medium has received a little attention. Mukhopadhyay and Layek (2008) presented the effects of variable viscosity through a porous medium over a stretching sheet in presence of thermal radiation.

Recently, fractional calculus has gained tremendous popularity among the researchers because of singular kernel with locality and non-singular kernel with non-locality problem. Caputo and Fabrizio used an exponential function in fractional derivative to avoid the singular kernel problem. Mirza and Vieru (2017) analyzed that the use of the time-fractional derivative without singular kernel is more advantageous than Caputo time-fractional derivative. Nehad et al. (2016) applied CF fractional derivatives to analyze the solutions for heat transfer of second grade fluids over vertical oscillating plates. "A comparative study has been obtained by using the $\mathrm{AB}$ and $\mathrm{CF}$ fractional derivatives for casson fluid model with chemical reaction and heat generation" by Sheikh et al. (2017). Few works using fractional derivatives have been studied in Histrov (2017), Atangana and Baleanu (2016). A comparative study of Atangana-Baleanu and Caputo-Fabrizio evaluation of fractional derivatives for heat and mass transfer of fluid over a vertical plate has been done by Khan et al. (2017).

The main objective of this paper is to investigate the effects of variable viscosity and thermal conductivity over a moving vertical plate in porous medium and comparing the results with the approaches $\mathrm{AB}$ and $\mathrm{CF}$ fractional derivatives. The non-dimensional governing equations with the non-dimensional boundary conditions are discretized with ordinary finite-difference kernel solved numerically with the help of $\mathrm{AB}$ and $\mathrm{CF}$ fractional derivative methods by developing suitable programming code in MATLAB. A comparative analysis under different parameters is represented graphically as well as in tabular form.

\section{Mathematical Formulation}

Consider a two-dimensional free convection steady heat and mass transfer flow of viscous incompressible electrically conducting fluid past a moving vertical plate in a porous medium (Figure 1). Here a uniform magnetic field is applied in a direction perpendicular to the fluid flow. The $\bar{x}$-axis is taken along the vertical plate in the direction of the flow and $\bar{y}$ axis is normal to it. At $t=0^{+}$, the fluid have gained the temperature $\bar{T}_{w}$, and concentration level near the plate is $\bar{C}_{w}$. The fluid properties are assumed to be constant except for the fluid viscosity and thermal conductivity which are assumed to vary as an inverse linear function of temperature. A uniform magnetic field of strength $B_{0}$ is applied normal to the plate. There is no chemical reaction between the fluid and diffusing species. Plate temperature $\bar{T}_{w}$ is variable and $\bar{T}_{\infty}$ is the free stream temperature assumed constant.

The variable viscosity and thermal conductivity are governed by the following equations under the boundary layer approximation: 
International Journal of Mathematical, Engineering and Management Sciences

Vol. 6, No. 2, 493-509, 2021

https://doi.org/10.33889/IJMEMS.2021.6.2.030

Equation of Continuity:

$$
\frac{\partial \bar{u}}{\partial \bar{x}}+\frac{\partial \bar{v}}{\partial \bar{y}}=0
$$

Equation of conservation of momentum:

$$
\frac{\partial \bar{u}}{\partial \bar{t}}+\bar{u} \frac{\partial \bar{u}}{\partial \bar{x}}+\bar{v} \frac{\partial \bar{u}}{\partial \bar{y}}=\vartheta \frac{\partial^{2} \bar{u}}{\partial \bar{y}^{2}}+\frac{1}{\rho} \frac{\partial \mu}{\partial \bar{y}} \frac{\partial \bar{u}}{\partial \bar{y}}-\frac{\sigma B_{0}^{2} \bar{u}}{\rho}+g \beta_{t}\left(\bar{T}-\bar{T}_{\infty}\right)+g \beta_{c}\left(\bar{C}-\bar{C}_{\infty}\right)-\frac{\vartheta}{\rho K} \bar{u}+\frac{\bar{F}_{m}}{\rho}
$$

Equation of conservation of energy:

$$
\rho C_{p}\left(\frac{\partial \bar{T}}{\partial \bar{t}}+\bar{u} \frac{\partial \bar{T}}{\partial \bar{x}}+\bar{v} \frac{\partial \bar{T}}{\partial \bar{y}}\right)=\frac{\partial \lambda}{\partial \bar{y}} \cdot \frac{\partial \bar{T}}{\partial \bar{y}}+\lambda \frac{\partial^{2} \bar{T}}{\partial \bar{y}^{2}}+\mu\left(\frac{\partial \bar{u}}{\partial \bar{y}}\right)^{2}-\frac{\partial \bar{q}_{r}}{\partial \bar{y}}
$$

Equation of concentration:

$$
\frac{\partial \bar{C}}{\partial \bar{t}}+\bar{u} \frac{\partial \bar{C}}{\partial \bar{x}}+\bar{v} \frac{\partial \bar{C}}{\partial \bar{y}}=\frac{\partial D_{m}}{\partial \bar{y}} \frac{\partial \bar{C}}{\partial \bar{y}}+D_{m} \frac{\partial^{2} \bar{C}}{\partial \bar{y}^{2}}
$$

The boundary conditions are:

$$
\begin{aligned}
& \bar{t} \leq 0: \bar{u}=0, \bar{T}=\bar{T}_{\infty}, \bar{C}=\bar{C}_{\infty} \forall y \\
& \bar{t}>0: \bar{u}=U_{0}, \bar{T}=\bar{T}_{w}=\bar{T}_{\infty}+b \bar{x}^{n}, \bar{C}=\bar{C}_{w}=\bar{C}_{\infty}+a \bar{x}^{m} \text { at } \bar{y}=0 \\
& \bar{t}>0: \bar{u} \rightarrow 0, \bar{T} \rightarrow \bar{T}_{\infty}, \bar{C} \rightarrow \bar{C}_{\infty} \text { at } \bar{y} \rightarrow \infty
\end{aligned}
$$

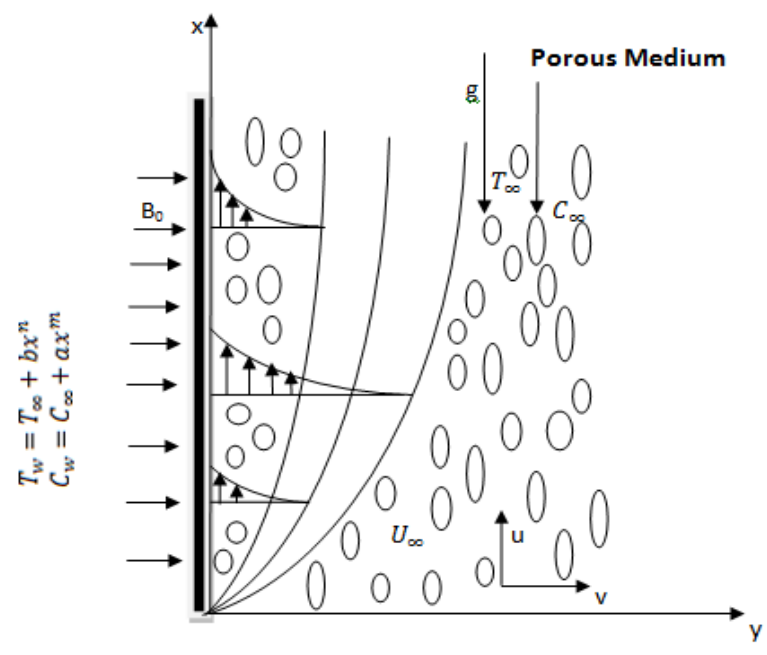

Figure 1. Schematic diagram of the flow. 
International Journal of Mathematical, Engineering and Management Sciences

Vol. 6, No. 2, 493-509, 2021

https://doi.org/10.33889/IJMEMS.2021.6.2.030

where, $\bar{u}$ and $\bar{v}$ are the fluid velocities in the direction of $\bar{x}$ and $\bar{y}$ respectively, $\vartheta$ is the kinematic viscosity, $\vartheta_{\infty}$ is the kinematic viscosity of the fluid in the free stream, $\rho$ is the fluid density, $\mu$ is the viscosity of the fluid, $\sigma$ is the electrical conductivity, $g$ is the acceleration due to gravity, $\beta_{t}$ is the volume expansion coefficient for heat transfer, $\beta_{c}$ is the volume expansion coefficient for mass transfer, $\bar{T}$ is the fluid temperature within the boundary layer, $\bar{T}_{\infty}$ is the temperature at free stream of the fluid, $\bar{C}$ is the species concentration of the fluid, $\bar{C}_{\infty}$ is the concentration at free stream of the fluid, $\lambda$ is the thermal conductivity of the fluid, $C_{p}$ is the specific heat at constant pressure, $\bar{q}_{r}$ is the radiative heat flux, $D_{m}$ is the mass diffusivity, $U_{0}$ is the velocity of the plate, $K$ is permeability of the porous medium, $\bar{J}$ is the electric current density, $\bar{a}$ is the mean absorption coefficient, $\bar{\sigma}$ is the Stefan - Boltzmann constant.

where, $\bar{F}_{m}=\vec{J} \times \vec{B}=\sigma[\vec{q} \times \vec{B}] \times \vec{B}=\sigma \bar{u} B_{0}{ }^{2}, \vec{q}$ be fluid velocity at a particular point, $\vec{B}=B_{0} \hat{j}$ be the applied magnetic field.

By Rosseland approximation,

The radiative heat flux, $\bar{q}_{r}=-\frac{4 \bar{\sigma}}{3 \bar{a}} \frac{\partial \bar{T}^{4}}{\partial \bar{y}}$

We expand $\bar{T}^{4}$ in Taylor's series about $\bar{T}_{\infty}$ as follows:

$\bar{T}^{4}=\bar{T}_{\infty}^{4}+4 \bar{T}_{\infty}^{3}\left(\bar{T}-\bar{T}_{\infty}\right)+6 \bar{T}_{\infty}^{2}\left(\bar{T}-\bar{T}_{\infty}\right)^{2}+$

By neglecting the higher order terms beyond $1^{\text {st }}$ degree in $\bar{T}-\bar{T}_{\infty}$ we have

$\bar{T}^{4}=4 \bar{T}_{\infty}^{3} \bar{T}-3 \bar{T}_{\infty}^{4}$

Using equations (6) and (7), equation (3) reduces to

$\rho C_{p}\left(\frac{\partial \bar{T}}{\partial \bar{t}}+\bar{u} \frac{\partial \bar{T}}{\partial \bar{x}}+\bar{v} \frac{\partial \bar{T}}{\partial \bar{y}}\right)=\frac{\partial \lambda}{\partial \bar{y}} \cdot \frac{\partial \bar{T}}{\partial \bar{y}}+\lambda \frac{\partial^{2} \bar{T}}{\partial \bar{y}^{2}}+\mu\left(\frac{\partial \bar{u}}{\partial \bar{y}}\right)^{2}-\frac{16 \bar{\sigma} T_{\infty}^{3}}{3 \bar{a}} \frac{\partial^{2} \bar{T}^{4}}{\partial \bar{y}^{2}}$

Appling the following non dimensional quantities:

$x=\frac{U_{0} \bar{x}}{\vartheta_{\infty}}, y=\frac{U_{0} \bar{y}}{\vartheta_{\infty}}, u=\frac{\bar{u}}{U_{0}}, v=\frac{\bar{v}}{U_{0}}, \theta=\frac{\bar{T}_{w}-\bar{T}}{\bar{T}_{w}-\bar{T}_{\infty}}, \phi=\frac{\bar{C}_{w}-\bar{C}}{\bar{C}_{w}-\bar{C}_{\infty}}, t=\frac{U_{0}{ }^{2} \bar{t}}{\vartheta_{\infty}}$

According to Lai and Kulacki (1990) the viscosity and thermal conductivity of the fluid are assumed to be inverse linear function of temperature as follows: 
International Journal of Mathematical, Engineering and Management Sciences

Vol. 6, No. 2, 493-509, 2021

https://doi.org/10.33889/IJMEMS.2021.6.2.030

$$
\begin{aligned}
& \frac{1}{\mu}=\frac{1}{\mu_{\infty}}\left\{1+\gamma\left(\bar{T}-\bar{T}_{\infty}\right)\right\} \\
& \frac{1}{\lambda}=\frac{1}{\lambda_{\infty}}\left\{1+\delta\left(\bar{T}-\bar{T}_{\infty}\right)\right\}
\end{aligned}
$$

where, $\gamma$ and $\delta$ are constants which depend on the thermal property of the fluid.

We define two parameters as, $\theta_{r}=\frac{\bar{T}_{w}-\bar{T}_{r}}{\bar{T}_{w}-\bar{T}_{\infty}}$ is called viscosity parameter and $\theta_{c}=\frac{\bar{T}_{w}-\bar{T}_{c}}{\bar{T}_{w}-\bar{T}_{\infty}}$ is called thermal Conductivity parameter.

Using these two parameters in (10) and (11), we have the viscosity and thermal conductivity respectively as

$$
\mu=\frac{\mu_{\infty}\left(1-\theta_{r}\right)}{\theta-\theta_{r}}, \lambda=\frac{\lambda_{\infty}\left(1-\theta_{c}\right)}{\theta-\theta_{c}}
$$

It is also important to note that $\theta_{r}$ is negative for liquid and positive for gases.

Using the transformations (9) and (12), the non-dimensional forms of (2), (3) and (4) are

$$
\begin{aligned}
& \frac{\partial u}{\partial t}+u \frac{\partial u}{\partial x}+v \frac{\partial u}{\partial y}=\left(\frac{1-\theta_{r}}{\theta-\theta_{r}}\right) \frac{\partial^{2} u}{\partial y^{2}}-\frac{1-\theta_{r}}{\left(\theta-\theta_{r}\right)^{2}} \frac{\partial \theta}{\partial y} \frac{\partial u}{\partial y} \\
&-M u+G r(1-\theta)+G r_{m}(1-\phi)-\left(\frac{1-\theta_{r}}{\theta-\theta_{r}}\right) u K_{p} \\
& \operatorname{Pr}\left(\frac{\partial \theta}{\partial t}+u \frac{\partial \theta}{\partial x}-\frac{n(1-\theta)}{x} u+v \frac{\partial \theta}{\partial y}\right)=\left(\frac{4}{3} K_{r}-\frac{1-\theta_{c}}{\theta-\theta_{c}}\right) \frac{\partial^{2} \theta}{\partial y^{2}}-\frac{1-\theta_{r}}{\left(\theta-\theta_{r}\right)^{2}}\left(\frac{\partial \theta}{\partial y}\right)^{2}+\left(\frac{1-\theta_{r}}{\theta-\theta_{r}}\right) \operatorname{Pr} \cdot E c\left(\frac{\partial u}{\partial y}\right)^{2} \\
& \frac{\partial \phi}{\partial t}+u \frac{\partial \phi}{\partial x}-\frac{m(1-\phi)}{x} u+v \frac{\partial \phi}{\partial y}=-\frac{1-\theta_{r}}{\left(\theta-\theta_{r}\right)^{2}} \cdot \frac{1}{S c} \frac{\partial \theta}{\partial y} \cdot \frac{\partial \phi}{\partial y}+\frac{1}{S c}\left(\frac{1-\theta_{c}}{\theta-\theta_{c}}\right) \frac{\partial^{2} \phi}{\partial y^{2}}
\end{aligned}
$$

The corresponding initial and boundary conditions are transformed to:

$$
\begin{aligned}
& t \leq 0: u=0, v=0, \theta=0, \phi=0 \quad \forall y \\
& t>0: u=1, \quad v=0, \theta=0, \phi=0 \quad \text { at } y=0 \\
& t>0: u \rightarrow 0, v \rightarrow 0, \theta \rightarrow 1, \phi \rightarrow 1 \text { at } y \rightarrow \infty
\end{aligned}
$$


International Journal of Mathematical, Engineering and Management Sciences

Vol. 6, No. 2, 493-509, 2021

https://doi.org/10.33889/IJMEMS.2021.6.2.030

where, $E c=\frac{U_{o}{ }^{2}}{\bar{R}_{w} C_{p}}$ is the Eckert number, $K_{p}=\frac{u_{\infty}{ }^{2}}{\rho K U_{0}{ }^{2}}$ is the Permeability parameter, $S c=\frac{\vartheta_{\infty}}{D_{m}} \quad$ is $\quad$ the $\quad$ Schmidt $\quad$ number, $M=\frac{\sigma B_{0}{ }^{2} \vartheta_{\infty}}{\rho U_{0}{ }^{2}} \quad$ is the Magnetic field parameter, $K r=\frac{16 \bar{a} \vartheta_{\infty} \bar{\sigma} \bar{T}_{\infty}^{2}}{3 \lambda_{\infty} U_{0}{ }^{2}}$ is the radiation parameter, $G r=\frac{\vartheta_{\infty} g \beta_{t}\left(\bar{T}_{w}-\bar{T}_{\infty}\right)}{U_{0}{ }^{3}}$ is the Grashof Number, $G r_{m}=\frac{\vartheta_{\infty} g \beta_{c}\left(\bar{C}_{w}-\bar{C}_{\infty}\right)}{U_{0}{ }^{3}}$ is the Concentration buoyancy parameter and $\operatorname{Pr}=\frac{\rho \vartheta_{\infty} C_{p}}{\lambda_{\infty}}$ is the Prandlt number.

\subsection{Atangana-Baleanu (AB) Fractional Derivatives}

To express $\mathrm{AB}$ fractional approach, "The governing partial differential equations can be written with respect to time by the $\mathrm{AB}$ fractional operator of the order $0<\alpha<1$ ", equations (13)-(15) become

$$
\begin{aligned}
A B\left(\frac{\partial^{\alpha} u(y, t)}{\partial t^{\alpha}}\right)= & -u \frac{\partial u}{\partial x}-v \frac{\partial u}{\partial y}+\left(\frac{1-\theta_{r}}{\theta-\theta_{r}}\right) \frac{\partial^{2} u}{\partial y^{2}}-\frac{1-\theta_{r}}{\left(\theta-\theta_{r}\right)^{2}} \frac{\partial \theta}{\partial y} \frac{\partial u}{\partial y} K_{p} \\
& -M u+G r(1-\theta)+G r_{m}(1-\phi)-\left(\frac{1-\theta_{r}}{\theta-\theta_{r}}\right) u \\
A B\left(\frac{\partial^{\alpha} \theta(y, t)}{\partial t^{\alpha}}\right)= & -u \frac{\partial \theta}{\partial x}-v \frac{\partial \theta}{\partial y}+\frac{n(1-\theta)}{x} u+\frac{1}{\operatorname{Pr}}\left(\frac{4}{3} K r-\frac{1-\theta_{c}}{\theta-\theta_{c}}\right) \frac{\partial^{2} \theta}{\partial y^{2}}-\frac{1}{\operatorname{Pr}} \frac{1-\theta_{r}}{\left(\theta-\theta_{r}\right)^{2}}\left(\frac{\partial \theta}{\partial y}\right)^{2}+\left(\frac{1-\theta_{r}}{\theta-\theta_{r}}\right) \cdot E c\left(\frac{\partial u}{\partial y}\right)^{2} \\
A B\left(\frac{\partial^{\alpha} \phi(y, t)}{\partial t^{\alpha}}\right)= & -u \frac{\partial \phi}{\partial x}-v \frac{\partial \phi}{\partial y}+\frac{m(1-\phi)}{x} u-\frac{1-\theta_{r}}{\left(\theta-\theta_{r}\right)^{2}} \cdot \frac{1}{S c} \frac{\partial \theta}{\partial y} \cdot \frac{\partial \phi}{\partial y}+\frac{1}{S c}\left(\frac{1-\theta_{c}}{\theta-\theta_{c}}\right) \frac{\partial^{2} \phi}{\partial y^{2}}
\end{aligned}
$$

where, $\frac{\partial^{\alpha} u(y, t)}{\partial t^{\alpha}}$ is the $\mathrm{AB}$ fractional operator of order $\alpha$ defined as

$A B\left(\frac{\partial^{\alpha} u(y, t)}{\partial t^{\alpha}}\right)=\frac{1}{1-\alpha} \int_{0}^{t} u^{\prime}(y, t) E_{\alpha}\left(\frac{-\alpha(z-t)}{1-\alpha}\right) d t$

and $E_{\alpha}\left(-t^{\alpha}\right)=\sum_{m=0}^{\infty} \frac{(-t)^{\alpha m}}{\Gamma(1+\alpha m)}$ is the Mittag-Leffler function.

\subsection{Caputo-Fabrizio (CF) Fractional Derivatives}

To express Caputo-Fabrizio fractional derivatives approach, "The governing partial differential equations can be written with respect to time by the $\mathrm{CF}$ fractional operator of the 
order $0<\beta<1$ ", equations (13)-(15) become

$$
\begin{aligned}
& C F\left(\frac{\partial^{\beta} u(y, t)}{\partial t^{\beta}}\right)=-u \frac{\partial u}{\partial x}-v \frac{\partial u}{\partial y}+\left(\frac{1-\theta_{r}}{\theta-\theta_{r}}\right) \frac{\partial^{2} u}{\partial y^{2}}-\frac{1-\theta_{r}}{\left(\theta-\theta_{r}\right)^{2}} \frac{\partial \theta}{\partial y} \frac{\partial u}{\partial y}-M u \\
&+G r(1-\theta)+G r_{m}(1-\phi)-\left(\frac{1-\theta_{r}}{\theta-\theta_{r}}\right) u K_{p} \\
& C F\left(\frac{\partial^{\beta} \theta(y, t)}{\partial t^{\beta}}\right)=-u \frac{\partial \theta}{\partial x}-v \frac{\partial \theta}{\partial y}+\frac{n(1-\theta)}{x} u+\frac{1}{\operatorname{Pr}}\left(\frac{4}{3} K r-\frac{1-\theta_{c}}{\theta-\theta_{c}}\right) \frac{\partial^{2} \theta}{\partial y^{2}}-\frac{1}{\operatorname{Pr}} \frac{1-\theta_{r}}{\left(\theta-\theta_{r}\right)^{2}}\left(\frac{\partial \theta}{\partial y}\right)^{2}+\left(\frac{1-\theta_{r}}{\theta-\theta_{r}}\right) \cdot E c\left(\frac{\partial u}{\partial y}\right)^{2} \\
& C F\left(\frac{\partial^{\beta} \phi(y, t)}{\partial t^{\beta}}\right)=-u \frac{\partial \phi}{\partial x}-v \frac{\partial \phi}{\partial y}+\frac{m(1-\phi)}{x} u-\frac{1-\theta_{r}}{\left(\theta-\theta_{r}\right)^{2}} \cdot \frac{1}{S c} \frac{\partial \theta}{\partial y} \cdot \frac{\partial \phi}{\partial y}+\frac{1}{S c}\left(\frac{1-\theta_{c}}{\theta-\theta_{c}}\right) \frac{\partial^{2} \phi}{\partial y^{2}}
\end{aligned}
$$

where, $\frac{\partial^{\beta} u(y, t)}{\partial t^{\beta}}$ is the CF fractional operator of order $\beta$ defined as

$$
C F\left(\frac{\partial^{\beta} u(y, t)}{\partial t^{\beta}}\right)=\frac{1}{1-\beta} \int_{0}^{t} u^{\prime}(y, t) \operatorname{Exp}\left(\frac{-\beta(z-t)}{1-\beta}\right) d t
$$

\section{Solution of the Problem}

Solutions of equations (17) - (20) or (21) - (24) are obtained by using ordinary finite difference scheme. Discritization is performed using the following formulae:

$$
\frac{\partial u}{\partial t}=\frac{u_{i+1, j, k}-u_{i, j, k}}{\Delta t}, \frac{\partial u}{\partial x}=\frac{u_{i, j+1, k}-u_{i, j, k}}{\Delta x}, \frac{\partial u}{\partial y}=\frac{u_{i, j, k+1}-u_{i, j, k}}{\Delta y}, \frac{\partial^{2} u}{\partial y^{2}}=\frac{u_{i, j, k+1}-2 u_{i, j, k}+u_{i, j, k-1}}{\Delta y^{2}}
$$

etc.

The fractional derivatives given by (20) or (24) are calculated using numerical integration. Finally the set of equations (17) - (19) or (21) - (23) together with boundary condition (16) completely discritized and the discritized equations are solved by using an iterative method based on Gaussseidel scheme.

The boundary conditions given in equation (16), now reduces to the form:

$$
\begin{aligned}
& t \leq 0: u_{i, j, k}=0, v_{i, j, k}=0, \theta_{i, j, k}=0, \phi_{i, j, k}=0 \quad \forall i, j, k \\
& t>0: u_{i, j, 1}=1, v_{i, j, 1}=0, \theta_{i, j, 1}=0, \phi_{i, j, 1}=0 \text { since } k=1 \text { when } y=0 ; \\
& t>0: u_{i, j, N} \rightarrow 0, v_{i, j, N} \rightarrow 0, \theta_{i, j, N} \rightarrow 1, \phi_{i, j, N} \rightarrow 1 \text { since } y \rightarrow \infty, \text { means } k \rightarrow N .
\end{aligned}
$$

\subsection{Important Physical Parameters}

The physical parameter Skin-friction coefficient indicates physical wall shear stress, Nusselt number indicates physical rate of heat transfer and Sherwood number indicates mass transfer. 
International Journal of Mathematical, Engineering and Management Sciences

Vol. 6, No. 2, 493-509, 2021

https://doi.org/10.33889/IJMEMS.2021.6.2.030

\section{(a) Coefficient of Skin Friction}

By the Newton's law of viscosity:

(Viscous drag) $\left.\left.\tau=\mu \frac{\partial \bar{u}}{\partial \bar{y}}\right)_{\bar{y}=0}=\frac{\mu U_{0}^{2}}{y \vartheta_{\infty}} \frac{\partial u}{\partial y}\right)_{y=0}$.

The non-dimensional skin-friction is given by,

$$
\left.C_{f}=\frac{\tau}{\rho U_{0}^{2}}=\frac{\theta_{r}-1}{\theta_{r}} \cdot \frac{\partial u}{\partial y}\right)_{y=0} .
$$

\section{(b) Nusselt Number}

By the Fourier's law of heat conduction in the form:

(Heat flux) $\left.q=-\lambda \frac{\partial \bar{T}}{\partial \bar{y}}\right)_{\bar{y}=0}$.

The rate of heat transfer coefficient is given by Nusselt Number

$$
\left.N u=\frac{x q}{\lambda\left(\bar{T}_{w}-\bar{T}_{\infty}\right)}=-\frac{\theta_{c}-1}{\theta_{c}} \frac{\partial \theta}{\partial y}\right)_{y=0} .
$$

\section{(c) Sherwood Number}

The mass flux $q_{m}$ from the plate to the fluid is given by the Fick's law,

$$
\left.q_{m}=-D_{m} \frac{\partial \bar{C}}{\partial \bar{y}}\right)_{\bar{y}=0}
$$

The rate of mass transfer is given by Sherwood number

$$
\left.S h=\frac{x q_{m}}{D_{m}\left(\bar{C}_{w}-\bar{C}_{\infty}\right)}=-\left(\frac{\theta_{r}-1}{\theta_{r}}\right) \frac{\partial \phi}{\partial y}\right)_{y=0} .
$$

\section{Results and Discussion}

By applying non dimensional quantities, the non dimensional discretized governing equations along with the non-dimensional boundary conditions are solved with the help of $\mathrm{AB}$ and $\mathrm{CF}$ fractional derivative methods by developing suitable programming code in MATLAB using finite difference scheme. This analysis has been done to study the effects of various parameters such as $\theta_{r}, \theta_{c}, M, K r, S c, E c, \operatorname{Pr}$ etc. on velocity $(u)$, temperature $(\theta)$ and species concentration $(\phi)$ profiles in presence of time. The numerical results are shown graphically in Figure 2 to 
International Journal of Mathematical, Engineering and Management Sciences

Vol. 6, No. 2, 493-509, 2021

https://doi.org/10.33889/IJMEMS.2021.6.2.030

Figure 15. In the following discussion, the initial values of the parameters are considered as $\theta_{r}=-20, \theta_{c}=-12, M=1, \alpha=0.25, G r_{m}=0.1, G r=0.1, \beta=0.25, K r=0.05, S c=0.5, E c=0.1, \operatorname{Pr}=0.25, K_{p}=0.5$ unless otherwise stated.

\subsection{Graphical Representation}

In Figure 2, it is seen that with the increasing value of the Hartmann number, velocity decreases. The presence of magnetic field in the normal direction of the flow in an electrically conducting fluid produces Lorentz force which opposes the flow. To overcome this opposing force, some extra work should be done which is transformed to heat energy. Hence temperature increases (Figure 3). This is because that the applied magnetic field opposes the fluid motion and therefore enhancing the temperature for this response. With the increase of $M$ species concentration also increases (Figure 4).

Figure 5 depicts the distribution of velocity with the variation of the thermal conductivity parameter $\theta_{c}$. Velocity increases with the increasing value of $\theta_{c}$. In Figure 6 temperature decreases with the increasing value of $\theta_{c}$. Physically it means that as thermal conduction increases the transportation of heat from hot region to colder region increases. Since temperature within boundary layer is more than the outside so temperature is decreased. Again species concentration increases with the increasing value of $\theta_{c}$ (Figure 7).

The effects of viscosity parameter $\theta_{r}$ on velocity, temperature and species concentration distribution are plotted in Figure 8 to Figure 10. Figure 8 displays that dimensionless velocity decreases with the increase of $\theta_{r}$. This is due to the fact that with the increase of the viscosity parameter the thickness of the velocity boundary layer decreases. Physically, this is because of that a larger $\theta_{r}$ implies higher temperature difference between the fluid and the surface. Figure 9 shows that temperature increases with the increasing value of $\theta_{r}$. The viscosity causes a rise in the friction, when friction increases the area of the stretching surface in contact with the flow increases therefore generated heat from the friction on the surface is transferred to the flow which leads to a rise in the surface temperature and the flow is heated. The species concentration decreases for increasing value of $\theta_{r}$ (Figure 10).

The Eckert number $E c$ signifies the viscous dissipation of the fluid, on temperature it is plotted in Figure 11. It is seen that an increase in viscous dissipation of the fluid tends to increase in fluid temperature with increase of $E c$. In Figure 12, it is noticed that with the increase of radiation parameter $\mathrm{Kr}$ temperature increases. This is due to the fact that the thermal boundary layer thickness increases with the increase of $K r$ and hence temperature. Velocity decreases with the increasing value of Prandtl number $\operatorname{Pr}$ (Figure 13). This is due to the fact that with the increase of $P r$, viscosity increases, so velocity decreases. In Figure 14, it is noticed that with the increasing value of $\operatorname{Pr}$ temperature of the fluid decreases. For higher Prandtl number the fluid has a relatively high thermal conductivity which decreases the temperature. 
International Journal of Mathematical, Engineering and Management Sciences

Vol. 6, No. 2, 493-509, 2021

https://doi.org/10.33889/IJMEMS.2021.6.2.030

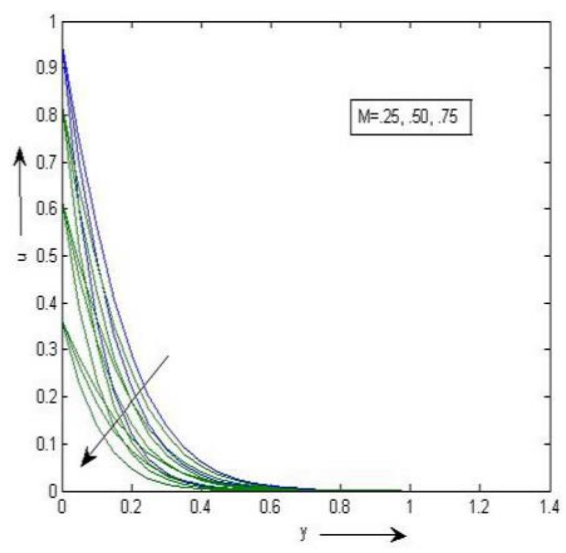

Figure 2. Effect of $M$ on velocity.

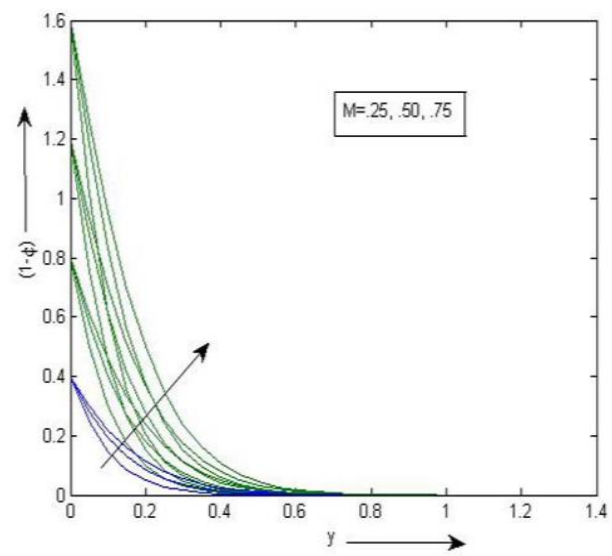

Figure 4. Effect of $M$ on concentration.

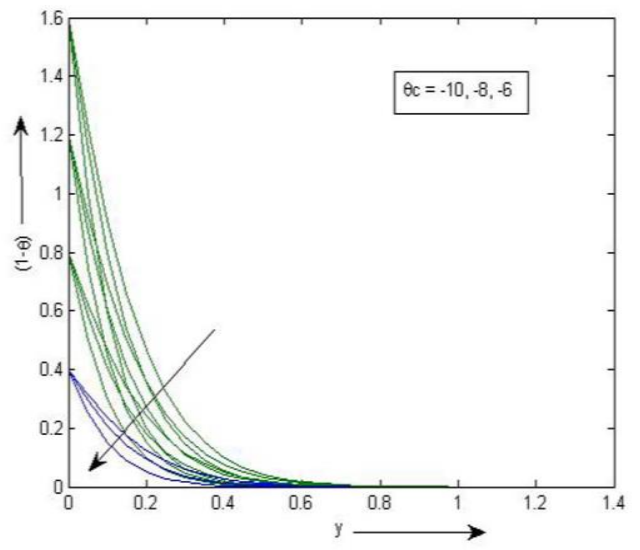

Figure 6. Effect of $\theta_{c}$ on temperature.

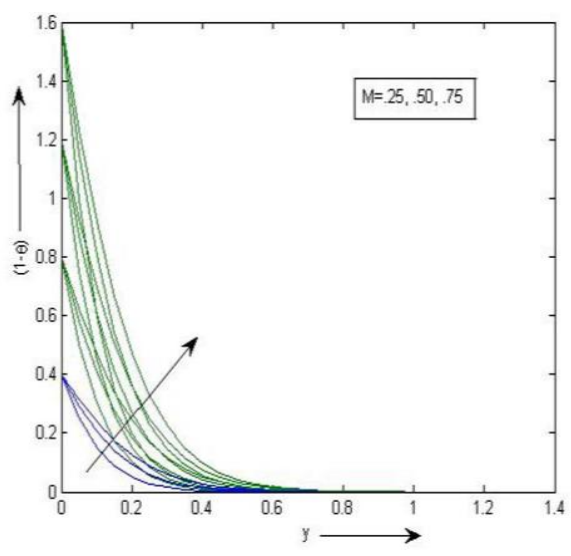

Figure 3. Effect of $M$ on temperature.

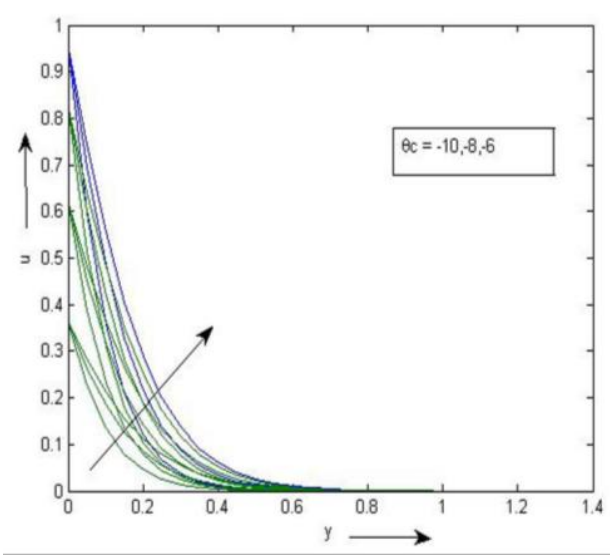

Figure 5. Effect of $\theta_{c}$ on velocity.

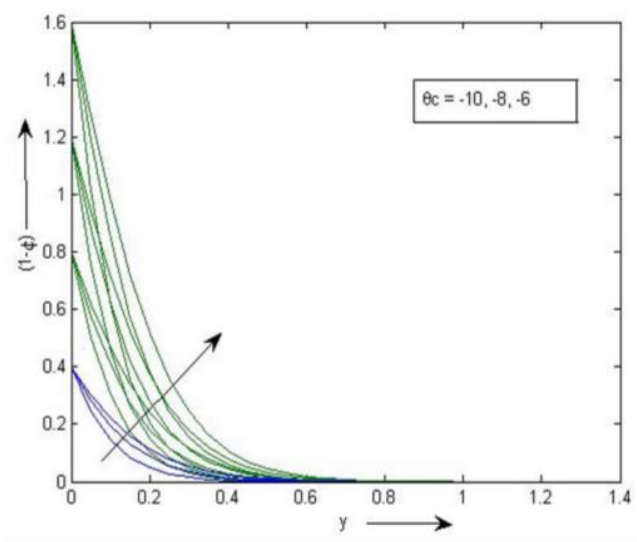

Figure 7. Effect of $\theta_{c}$ on concentration. 
International Journal of Mathematical, Engineering and Management Sciences

Vol. 6, No. 2, 493-509, 2021

https://doi.org/10.33889/IJMEMS.2021.6.2.030

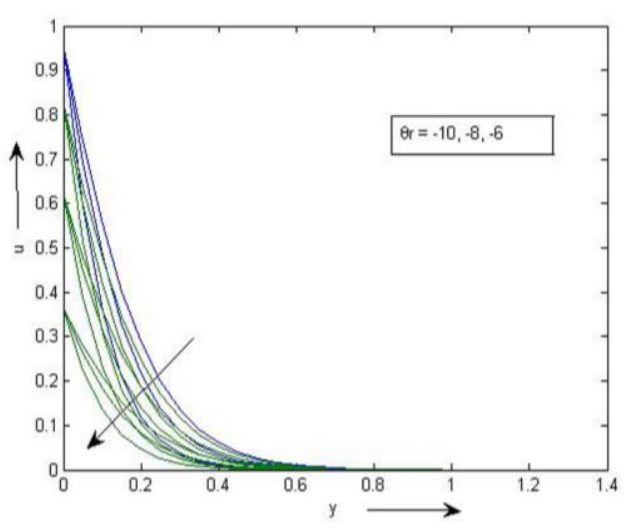

Figure 8. Effect of $\theta_{r}$ on velocity

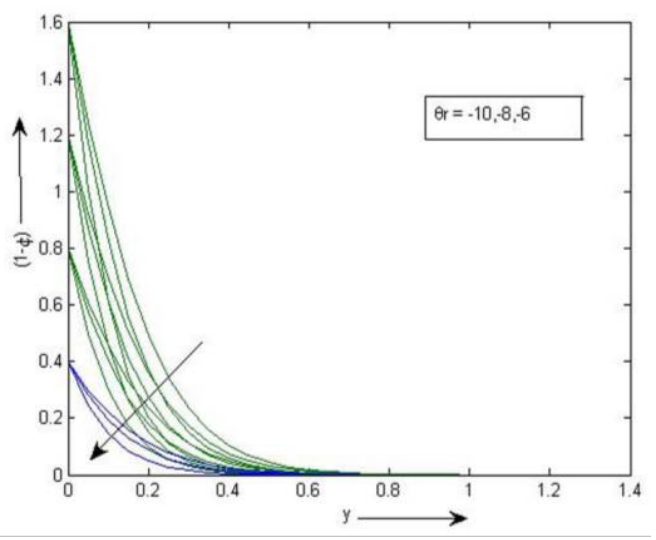

Figure 10. Effect of $\theta_{r}$ on concentration.

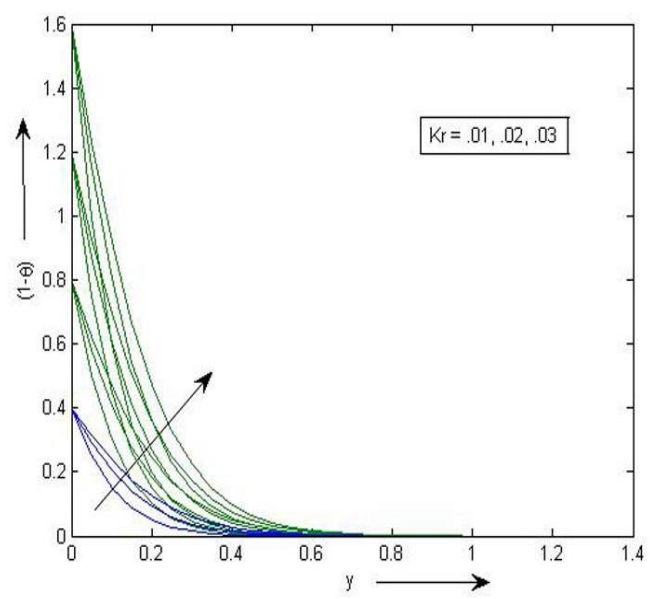

Figure 12. Effect of $K r$ on temperature.

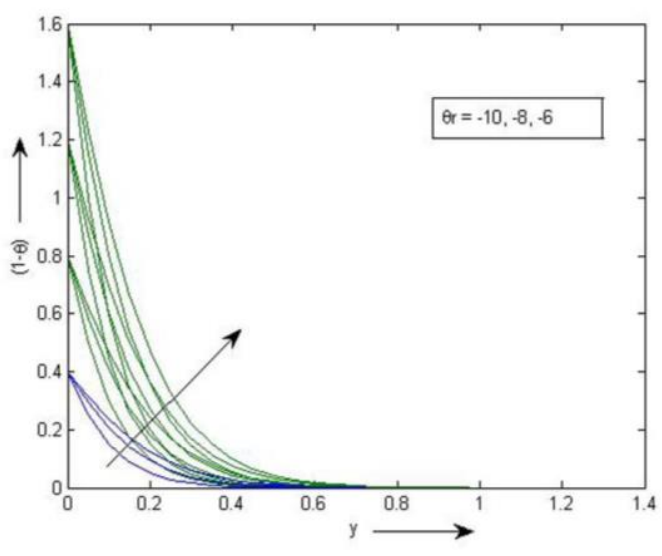

Figure 9. Effect of $\theta_{r}$ on temperature.

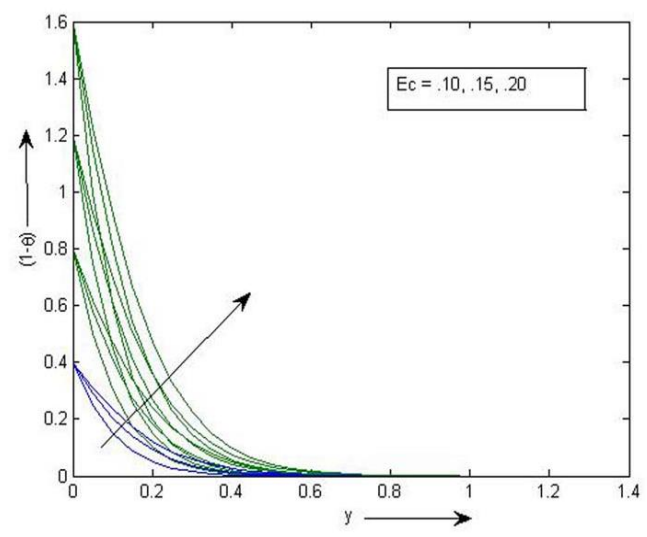

Figure 11. Effect of $E c$ on temperature.

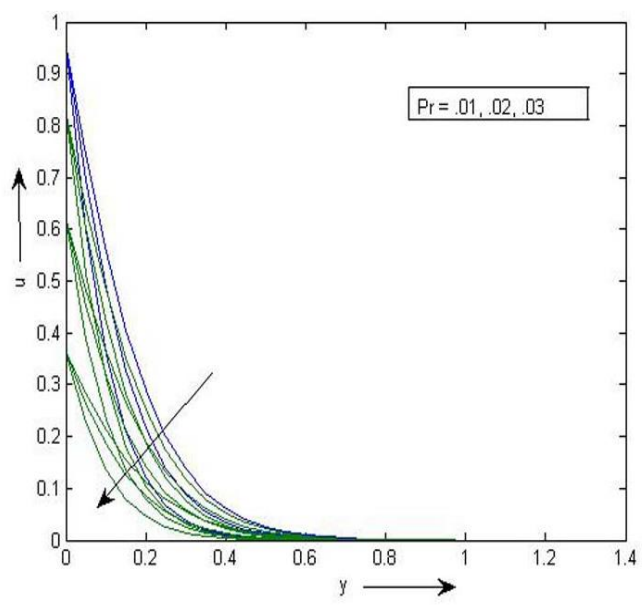

Figure 13. Effect of $\operatorname{Pr}$ on velocity. 


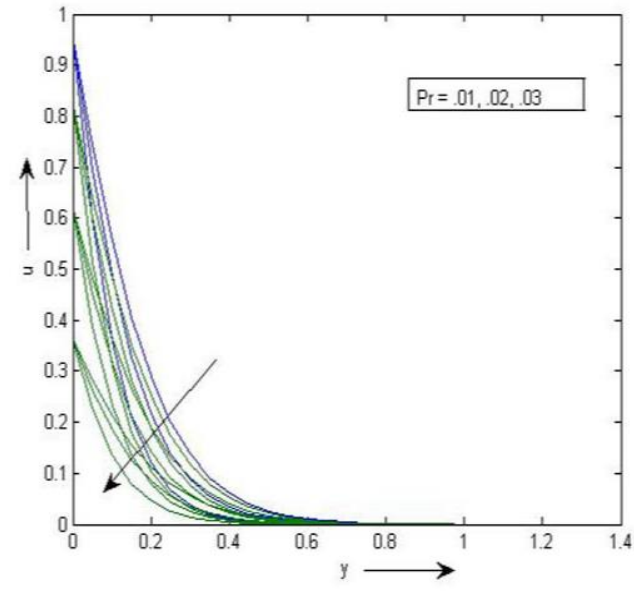

Figure 14. Effect of $\operatorname{Pr}$ on temperature.

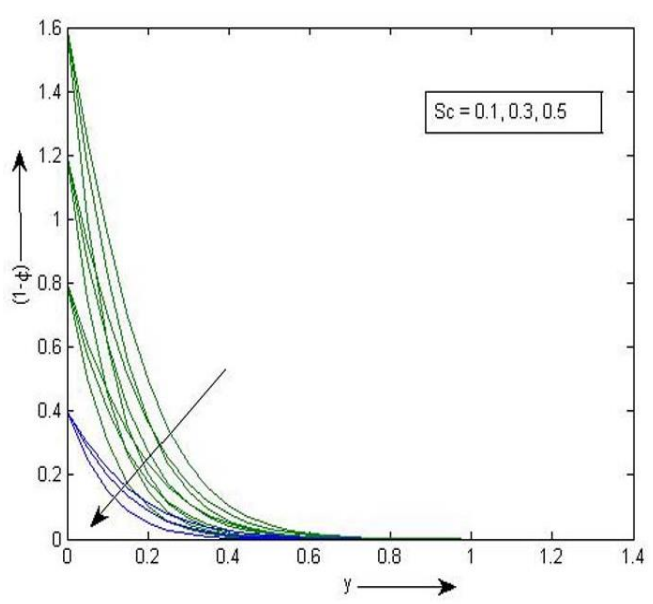

Figure 15. Effect of $S c$ on concentration.

In Figure 15, Sc is increased the concentration boundary layer becomes thinner than the viscous boundary layer, as a result of which velocity reduces. With thinner concentration boundary layer the concentration gradients are enhanced causing a decrease in concentration of species in the boundary layer.

\subsection{Comparision of $\mathrm{AB}$ and $\mathrm{CF}$ Fractional Derivatives for Various Values of the Parameters in Tabular Form}

Here we compare between $\mathrm{AB}$ and $\mathrm{CF}$ fractional derivative for various values of the parameters $M, \theta_{c}, \theta_{r}, S c, K r, \operatorname{Pr}, E c$ taking $y=0.4$ and $t=0.4$. From the following tables it is found that the values of the velocity, temperature and concentration profiles for various parameters are almost the same for both the methods- $\mathrm{AB}$ and $\mathrm{CF}$ fractional derivative.

Table 1. Effect of $M$ on $u, \theta$ and $\phi$.

\begin{tabular}{|c|c|c|c|c|c|c|c|c|}
\hline \multirow{2}{*}{$\boldsymbol{M}$} & \multirow{2}{*}{$y$} & \multirow{2}{*}{$t$} & \multicolumn{2}{|c|}{$u$} & \multicolumn{2}{|c|}{$\theta$} & \multicolumn{2}{|c|}{$\phi$} \\
\hline & & & $\mathbf{A B}$ & CF & $\mathbf{A B}$ & $\mathbf{C F}$ & $\mathbf{A B}$ & CF \\
\hline \multirow{4}{*}{0.25} & \multirow{4}{*}{0.4} & 0.4 & 0.036610 & 0.036588 & 0.008659 & 0.008654 & 0.008459 & 0.008439 \\
\hline & & 0.8 & 0.060779 & 0.060758 & 0.017286 & 0.017204 & 0.017143 & 0.017140 \\
\hline & & 1.2 & 0.079966 & 0.079979 & 0.025779 & 0.025793 & 0.025928 & 0.025937 \\
\hline & & 1.6 & 0.092290 & 0.092380 & 0.034333 & 0.034418 & 0.034799 & 0.034889 \\
\hline \multirow{4}{*}{0.50} & \multirow{4}{*}{0.4} & 0.4 & 0.021852 & 0.021691 & 0.023380 & 0.023378 & 0.021848 & 0.021838 \\
\hline & & 0.8 & 0.036248 & 0.036159 & 0.046098 & 0.046054 & 0.045382 & 0.045347 \\
\hline & & 1.2 & 0.047737 & 0.047815 & 0.068543 & 0.068600 & 0.069422 & 0.069547 \\
\hline & & 1.6 & 0.054954 & 0.054961 & 0.090805 & 0.090810 & 0.094434 & 0.094528 \\
\hline \multirow{4}{*}{0.75} & \multirow{4}{*}{0.4} & 0.4 & 0.008157 & 0.008086 & 0.039695 & 0.039675 & 0.035782 & 0.035764 \\
\hline & & 0.8 & 0.013610 & 0.013544 & 0.077713 & 0.077694 & 0.075483 & 0.075427 \\
\hline & & 1.2 & 0.017888 & 0.017895 & 0.114888 & 0.114894 & 0.116372 & 0.116454 \\
\hline & & 1.6 & 0.020577 & 0.020582 & 0.150742 & 0.151763 & 0.159960 & 0.159988 \\
\hline
\end{tabular}


International Journal of Mathematical, Engineering and Management Sciences

Vol. 6, No. 2, 493-509, 2021

https://doi.org/10.33889/IJMEMS.2021.6.2.030

Table 2. Effect of $\theta_{c}$ on $u, \theta$ and $\phi$.

\begin{tabular}{|c|c|c|c|c|c|c|c|c|}
\hline \multirow{2}{*}{$\theta_{c}$} & \multirow{2}{*}{$y$} & \multirow{2}{*}{$t$} & \multicolumn{2}{|c|}{$u$} & \multicolumn{2}{|c|}{$\theta$} & \multicolumn{2}{|c|}{$\phi$} \\
\hline & & & AB & CF & $\mathbf{A B}$ & $\mathrm{CF}$ & $\mathbf{A B}$ & $\mathrm{CF}$ \\
\hline \multirow{4}{*}{-10} & \multirow{4}{*}{0.4} & 0.4 & 0.020589 & 0.020403 & 0.008648 & 0.008632 & 0.008443 & 0.008443 \\
\hline & & 0.8 & 0.017770 & 0.017698 & 0.017177 & 0.017165 & 0.017163 & 0.017163 \\
\hline & & 1.2 & 0.013406 & 0.013540 & 0.025657 & 0.025702 & 0.025982 & 0.025983 \\
\hline & & 1.6 & 0.007996 & 0.008025 & 0.034096 & 0.034181 & 0.034885 & 0.034886 \\
\hline \multirow{4}{*}{-8} & \multirow{4}{*}{0.4} & 0.4 & 0.054677 & 0.054514 & 0.023351 & 0.023349 & 0.021920 & 0.021909 \\
\hline & & 0.8 & 0.047369 & 0.047246 & 0.045914 & 0.045929 & 0.045696 & 0.045661 \\
\hline & & 1.2 & 0.035772 & 0.035882 & 0.068166 & 0.068223 & 0.070127 & 0.070152 \\
\hline & & 1.6 & $\begin{array}{l}0.021391 \\
\end{array}$ & 0.021530 & 0.089819 & 0.090224 & 0.095677 & 0.095679 \\
\hline \multirow{4}{*}{-6} & \multirow{4}{*}{0.4} & 0.4 & 0.091709 & 0.091658 & 0.039482 & 0.039462 & 0.036065 & 0.036048 \\
\hline & & 0.8 & 0.079513 & 0.079426 & 0.077050 & 0.077030 & 0.076657 & 0.076602 \\
\hline & & 1.2 & 0.060184 & 0.060362 & 0.113818 & 0.113824 & 0.118971 & 0.118973 \\
\hline & & 1.6 & 0.036088 & 0.036364 & 0.148869 & 0.149960 & 0.164496 & 0.164498 \\
\hline
\end{tabular}

Table 3. Effect of $\theta_{r}$ on $u, \theta$ and $\phi$.

\begin{tabular}{|c|c|c|c|c|c|c|c|c|}
\hline \multirow{2}{*}{$\theta_{r}$} & \multirow{2}{*}{$y$} & \multirow{2}{*}{$t$} & \multicolumn{2}{|c|}{$u$} & \multicolumn{2}{|c|}{$\theta$} & \multicolumn{2}{|c|}{$\phi$} \\
\hline & & & AB & CF & $\mathbf{A B}$ & $\mathbf{C F}$ & $\mathbf{A B}$ & CF \\
\hline \multirow{4}{*}{-10} & \multirow{4}{*}{0.4} & 0.4 & 0.090985 & 0.090834 & 0.008659 & 0.008654 & 0.035576 & 0.035559 \\
\hline & & 0.8 & 0.078321 & 0.078234 & 0.017286 & 0.017204 & 0.074725 & 0.074669 \\
\hline & & 1.2 & 0.058894 & 0.059070 & 0.025778 & 0.025823 & 0.114768 & 0.114848 \\
\hline & & 1.6 & 0.035137 & 0.035411 & 0.034233 & 0.034318 & 0.157360 & 0.157378 \\
\hline \multirow{4}{*}{-8} & \multirow{4}{*}{0.4} & 0.4 & 0.054312 & 0.054249 & 0.023378 & 0.023376 & 0.021777 & 0.021766 \\
\hline & & 0.8 & 0.046928 & 0.046805 & 0.046082 & 0.046048 & 0.045128 & 0.045093 \\
\hline & & 1.2 & 0.035492 & 0.035500 & 0.068537 & 0.068594 & 0.068887 & 0.068889 \\
\hline & & 1.6 & 0.021233 & 0.021270 & 0.090901 & 0.090906 & 0.093557 & 0.093559 \\
\hline \multirow{4}{*}{-6} & \multirow{4}{*}{0.4} & 0.4 & 0.020453 & 0.020367 & 0.039682 & 0.039562 & 0.008440 & 0.008430 \\
\hline & & 0.8 & 0.017710 & 0.017637 & 0.077489 & 0.077469 & 0.017120 & 0.017110 \\
\hline & & 1.2 & 0.013389 & 0.013393 & 0.114883 & 0.114890 & 0.025877 & 0.025887 \\
\hline & & 1.6 & 0.007996 & 0.007997 & 0.150628 & 0.151718 & 0.034688 & 0.034689 \\
\hline
\end{tabular}

Table 4. Effect of $K r$ and $E c$ on $\theta$.

\begin{tabular}{|c|c|c|c|c|c|c|c|c|c|}
\hline \multirow{2}{*}{$K r$} & \multirow{2}{*}{$y$} & \multirow{2}{*}{$t$} & \multicolumn{2}{|c|}{$\theta$} & \multirow{2}{*}{$E_{c}$} & \multirow{2}{*}{$y$} & \multirow{2}{*}{$t$} & \multicolumn{2}{|c|}{$\theta$} \\
\hline & & & $\mathbf{A B}$ & $\mathbf{C F}$ & & & & $\mathbf{A B}$ & $\mathbf{C F}$ \\
\hline \multirow{4}{*}{0.01} & \multirow{4}{*}{0.4} & 0.4 & 0.008670 & 0.008654 & \multirow{4}{*}{0.10} & \multirow{4}{*}{0.4} & 0.4 & 0.009850 & 0.009755 \\
\hline & & 0.8 & 0.017286 & 0.017205 & & & 0.8 & 0.018486 & 0.018305 \\
\hline & & 1.2 & 0.025880 & 0.025924 & & & 1.2 & 0.025819 & 0.025824 \\
\hline & & 1.6 & 0.034536 & 0.034620 & & & 1.6 & 0.034203 & 0.034219 \\
\hline \multirow{4}{*}{0.02} & \multirow{4}{*}{0.4} & 0.4 & 0.023385 & 0.023379 & \multirow{4}{*}{0.15} & \multirow{4}{*}{0.4} & 0.4 & 0.023729 & 0.023627 \\
\hline & & 0.8 & 0.046097 & 0.046052 & & & 0.8 & 0.046189 & 0.046165 \\
\hline & & 1.2 & 0.046087 & 0.046092 & & & 1.2 & 0.068586 & 0.068593 \\
\hline & & 1.6 & 0.090834 & 0.090839 & & & 1.6 & 0.090826 & 0.090831 \\
\hline \multirow{4}{*}{0.03} & \multirow{4}{*}{0.4} & 0.4 & 0.039670 & 0.039581 & \multirow{4}{*}{0.20} & \multirow{4}{*}{0.4} & 0.4 & 0.040323 & 0.040293 \\
\hline & & 0.8 & 0.077601 & 0.077582 & & & 0.8 & 0.077999 & 0.077992 \\
\hline & & 1.2 & 0.114864 & 0.114869 & & & 1.2 & 0.115122 & 0.115129 \\
\hline & & 1.6 & 0.151733 & 0.151796 & & & 1.6 & 0.150832 & 0.151833 \\
\hline
\end{tabular}


International Journal of Mathematical, Engineering and Management Sciences

Vol. 6, No. 2, 493-509, 2021

https://doi.org/10.33889/IJMEMS.2021.6.2.030

Table 5. Effect of $\operatorname{Pr}$ on $u, \theta$ and $S c$ on $\phi$.

\begin{tabular}{|c|c|c|c|c|c|c|c|c|c|}
\hline \multirow{2}{*}{$P r$} & \multirow{2}{*}{$y$} & \multirow{2}{*}{$t$} & \multicolumn{2}{|c|}{$u$} & \multicolumn{2}{|c|}{$\theta$} & \multirow[b]{2}{*}{$S c$} & \multicolumn{2}{|c|}{$\phi$} \\
\hline & & & $\mathbf{A B}$ & CF & $\mathbf{A B}$ & CF & & $\mathbf{A B}$ & CF \\
\hline \multirow{4}{*}{0.01} & \multirow{4}{*}{0.4} & 0.4 & 0.091703 & 0.091657 & 0.039570 & 0.039551 & \multirow{4}{*}{0.1} & 0.036467 & 0.036446 \\
\hline & & 0.8 & 0.079509 & 0.079420 & 0.077501 & 0.077482 & & 0.075666 & 0.075610 \\
\hline & & 1.2 & 0.060472 & 0.060550 & 0.114864 & 0.114869 & & 0.115655 & 0.115737 \\
\hline & & 1.6 & 0.036469 & 0.036545 & 0.150733 & 0.151796 & & 0.157770 & 0.157788 \\
\hline \multirow{4}{*}{0.02} & \multirow{4}{*}{0.4} & 0.4 & 0.054574 & 0.054514 & 0.023375 & 0.023373 & \multirow{4}{*}{0.3} & 0.022380 & 0.022374 \\
\hline & & 0.8 & 0.047387 & 0.047244 & 0.046087 & 0.046052 & & 0.045518 & 0.045497 \\
\hline & & 1.2 & 0.047294 & 0.047299 & 0.046087 & 0.046092 & & 0.068746 & 0.068801 \\
\hline & & 1.6 & 0.021586 & 0.021595 & 0.090734 & 0.090740 & & 0.092652 & 0.092654 \\
\hline \multirow{4}{*}{0.03} & \multirow{4}{*}{0.4} & 0.4 & 0.020487 & 0.020403 & 0.008659 & 0.008654 & \multirow{4}{*}{0.5} & 0.008556 & 0.008556 \\
\hline & & 0.8 & 0.017770 & 0.017698 & 0.017386 & 0.017205 & & 0.017153 & 0.017153 \\
\hline & & 1.2 & 0.013476 & 0.013490 & 0.025880 & 0.025924 & & 0.025755 & 0.025756 \\
\hline & & 1.6 & 0.008922 & 0.008925 & 0.034316 & 0.034320 & & 0.034360 & 0.034361 \\
\hline
\end{tabular}

From Table 1, 2, 3, 4 and 5 we have found some significance regarding velocity, temperature and concentration under the parameters $M, \theta_{c}, \theta_{r}, S c, K r, E c$ and $\operatorname{Pr}$ for the approaches AB and $\mathrm{CF}$ fractional derivatives. It is observed that, if time is less than 1 , the velocity obtained using $\mathrm{AB}$ approach is greater than the velocity computed with $\mathrm{CF}$ approach. Otherwise if the time is just greater than 1 , the velocity obtained by $\mathrm{CF}$ is greater than the velocity obtained by $\mathrm{AB}$ approach. Again for temperature profile under the parameters the value obtained by $\mathrm{CF}$ approach just greater than $\mathrm{AB}$ approach but for concentration profile the value obtained by $\mathrm{AB}$ approach is just greater than $\mathrm{CF}$ approach.

\subsection{Comparison of $\mathrm{AB}$ and $\mathrm{CF}$ method for Coefficient of Skin Friction, Nusselt number and Sherwood Number}

We are considering the values of the $\mathrm{AB}$ operator $(\alpha)$ and the $\mathrm{CF}$ operator $(\beta)$ as 0.2 and 0.4 . The values of $C_{f}, N u$ and $S h$ are calculated by both the AB and $\mathrm{CF}$ methods for various values of the involved parameters. The Coefficient of skin- friction $\left(C_{f}\right)$, rate of mass transfer in terms of Sherwood number $(S h)$ and rate of heat transfer in terms of Nusselt number $(\mathrm{Nu})$ at the plate $y=0.4$ and time $t=0.4$ against $\theta_{r}, \theta_{c}$ and $M$ are demonstrated in Table 6 to Table 8 .

Table 6. Variation of physical quantities with viscosity parameter for

$$
M=1, \theta_{c}=-12, G r=0.51, G r_{m}=0.51, \operatorname{Pr}=0.70, E c=0.1, S c=0.5, S o=5 \text {. }
$$

\begin{tabular}{|c|c|c|c|c|c|c|c|}
\hline \multirow{2}{*}{$\alpha / \beta$} & \multirow{2}{*}{$\theta_{r}$} & \multicolumn{2}{|c|}{$C_{f}$} & \multicolumn{2}{c|}{$N u$} & \multicolumn{2}{c|}{$S h$} \\
\cline { 2 - 8 } & & $\mathbf{A B}$ & $\mathbf{C F}$ & $\mathbf{A B}$ & \multicolumn{1}{c|}{$\mathbf{C F}$} & $\mathbf{A B}$ & $\mathbf{C F}$ \\
\hline \multirow{4}{*}{0.2} & -10 & -14.510708 & -14.55779 & 7.248705 & 7.237226 & 14.670122 & 14.52998 \\
\cline { 2 - 8 } & -8 & -8.049324 & -8.887118 & 4.305106 & 4.112306 & 9.541315 & 9.072564 \\
\cline { 2 - 8 } & -6 & -6.164552 & -6.555832 & 3.308188 & 3.170818 & 7.654831 & 7.339991 \\
\cline { 2 - 8 } & -4 & -5.123131 & -5.583362 & 2.756970 & 2.646633 & 6.562650 & 6.282060 \\
\hline \multirow{4}{*}{0.4} & -10 & -9.621844 & -10.860686 & 5.258340 & 4.994447 & 11.819109 & 10.54274 \\
\cline { 2 - 8 } & -8 & -7.082974 & -7.413013 & 3.720947 & 3.560238 & 8.412463 & 8.058354 \\
\cline { 2 - 8 } & -6 & -5.562100 & -5.992445 & 3.002892 & 2.878940 & 7.760696 & 7.110674 \\
\cline { 2 - 8 } & -4 & -4.652398 & -4.937746 & 2.561298 & 2.459358 & 6.986970 & 6.284284 \\
\hline
\end{tabular}


International Journal of Mathematical, Engineering and Management Sciences

Vol. 6, No. 2, 493-509, 2021

https://doi.org/10.33889/IJMEMS.2021.6.2.030

Table 7. Variation of physical quantities with thermal conductivity parameter for $M=1, \theta_{r}=-12, G r=0.51, G r_{m}=0.51, \operatorname{Pr}=0.70, E c=0.1, S c=0.5, S o=5$

\begin{tabular}{|c|c|c|c|c|c|c|c|}
\hline \multirow{2}{*}{$\alpha / \beta$} & \multirow{2}{*}{$\theta_{c}$} & \multicolumn{2}{|c|}{$C_{f}$} & \multicolumn{2}{c|}{$N u$} & \multicolumn{2}{c|}{$S h$} \\
\cline { 3 - 8 } & & $\mathbf{A B}$ & $\mathbf{C F}$ & $\mathbf{A B}$ & $\mathbf{C F}$ & $\mathbf{A}$ & $\mathbf{C F}$ \\
\hline \multirow{4}{*}{0.2} & -10 & -14.71153 & -14.75877 & 7.209884 & 7.19839 & 14.75818 & 14.61820 \\
\cline { 2 - 8 } & -8 & -8.267272 & -8.713509 & 4.258720 & 4.06159 & 9.160136 & 9.060340 \\
\cline { 2 - 8 } & -6 & -6.306919 & -6.711279 & 3.238662 & 3.09560 & 7.817970 & 7.61941 \\
\cline { 2 - 8 } & -4 & -5.254050 & -5.533527 & 2.637452 & 2.51804 & 6.814178 & 6.56182 \\
\hline \multirow{5}{*}{0.4} & -10 & -9.721226 & -10.959933 & 5.235210 & 4.96659 & 11.88594 & 11.6206 \\
\cline { 2 - 8 } & -8 & -7.166104 & -7.502525 & 3.685785 & 3.52061 & 8.980200 & 8.43676 \\
\cline { 2 - 8 } & -6 & -5.073432 & -6.712769 & 2.945959 & 2.81547 & 7.669259 & 7.03681 \\
\cline { 2 - 8 } & -4 & -4.144791 & -5.841899 & 2.458716 & 2.34579 & 6.858733 & 6.18878 \\
\hline
\end{tabular}

Table 8. Variation of physical quantities with magnetic field parameter for

$$
\theta_{r}=-20, \theta_{c}=-12, G r=0.51, G r_{m}=0.51, \operatorname{Pr}=0.70, E c=0.1, S c=0.5, \text { So }=5 \text {. }
$$

\begin{tabular}{|c|c|c|c|c|c|c|c|}
\hline \multirow{3}{*}{$\alpha / \beta$} & \multirow{2}{*}{$M$} & \multicolumn{2}{|c|}{$C_{f}$} & \multicolumn{2}{c|}{$N u$} & \multicolumn{2}{c|}{$S h$} \\
\cline { 3 - 8 } & & $\mathbf{A B}$ & $\mathbf{C F}$ & $\mathbf{A B}$ & $\mathbf{C F}$ & $\mathbf{A B}$ & $\mathbf{C F}$ \\
\hline \multirow{4}{*}{0.2} & 0.4 & -14.72545 & -14.89264 & 7.248048 & 7.236569 & 14.80152 & 14.76111 \\
\cline { 2 - 8 } & 0.8 & -8.733683 & -8.881993 & 4.302752 & 4.108959 & 9.189046 & 9.110243 \\
\cline { 2 - 8 } & 1.2 & -6.700837 & -6.806939 & 3.303619 & 3.165336 & 7.424904 & 7.396263 \\
\cline { 2 - 8 } & 1.6 & -5.599457 & -5.678780 & 2.748759 & 2.636274 & 6.522696 & 6.324539 \\
\hline \multirow{4}{*}{0.4} & 0.4 & -10.67705 & -10.916454 & 5.257273 & 4.990260 & 10.94694 & 10.66938 \\
\cline { 2 - 8 } & 0.8 & -7.533452 & -7.671426 & 3.718370 & 3.556140 & 8.120591 & 8.058800 \\
\cline { 2 - 8 } & 1.2 & -6.080710 & -6.720423 & 2.997566 & 2.871148 & 7.896560 & 7.237765 \\
\cline { 2 - 8 } & 1.6 & -5.213385 & -5.908385 & 2.551771 & 2.444825 & 6.502341 & 6.490837 \\
\hline
\end{tabular}

In Table 6, it is seen that coefficient of Skin- friction $\left(C_{f}\right)$ increases but $N u$ and $S h$ decreases for the increasing values of $\theta_{r}$. Physically negative values of $C_{f}$ mean that the surface exerts a drag force on the fluid. In Table 7, it is seen that $C_{f}$ increases but $N u$ and $S h$ decreases for the increasing values of $\theta_{c}$. It is because viscosity decreases with increasing thermal conductivity which enhances the magnitude of surface velocity gradient and reduces the magnitude of the heat transfer rate. Therefore skin-friction increases and Nusselt number and Sherwood number decreases with increasing $\theta_{c}$. In Table 8, it is seen that $C_{f}$ increases but $N u$ and $S h$ decreases for the increasing values of $M$. After comparison we observed that the values of skin-friction and Sherwood number using $\mathrm{CF}$ approach is slightly greater than $\mathrm{AB}$ approach. But for Nusselt number values of $\mathrm{AB}$ approach is just greater than $\mathrm{CF}$ approach.

\section{Conclusion}

In this study, the effects of variable viscosity and thermal conductuvity on MHD flow over a moving vertical plate in a porous medium with radiation and viscous dissipation is investigated. Based on the above analysis we can conclude that:

- Velocity, temperature and species concentration increases with the increasing value of $\mathrm{AB}$ fractional $(\alpha)$ parameter and $\mathrm{CF}$ fractional parameter $(\beta)$. 
International Journal of Mathematical, Engineering and Management Sciences

Vol. 6, No. 2, 493-509, 2021

https://doi.org/10.33889/IJMEMS.2021.6.2.030

- Magnetic field and viscosity have retarding effect in the flow.

- Increasing value of Magnetic field parameter decreases the value of velocity but increases the values of temperature and species concentration.

- When the viscosity parameter increases, the velocity and the species concentration decreases whereas temperature increases.

- With the increasing thermal conductivity parameter, the velocity and the species concentration increases but the temperature decreases.

- Velocity and temperature increases with the increasing value of eckert number and radiation parameter.

- Velocity and temperature increases with the increasing value of prandlt number. Temperature and species concentration decrease with the increasing value of the Schmidt number.

- The values of the velocity, temperature and concentration profiles for various parameters are almost the same for both the methods- $\mathrm{AB}$ and $\mathrm{CF}$ fractional derivatives. As gamma function is present inside the exponential function in $\mathrm{AB}$ fractional derivative method, so the result obtained by it is more accurate over the CF fractional derivative method.

\section{Conflict of Interest}

The authors confirm that there is no conflict of interest to declare for this publication.

\section{Acknowledgement}

The authors express their sincere thanks to the referees and the editor for their valuable comments and suggestions towards the improvement of the paper.

\section{References}

Atangana, A., \& Baleanu, D. (2016). New fractional derivatives with nonlocal and non-singular kernel: Theory and application to heat transfer model. Thermal Science, 20(2), 763-769.

Aldoss, T.K., Al-Nimr, M.A., Jarrah, M.A., \& Al-Sha'er, B.J. (1995). Magnetohydrodynamic mixed convection from a vertical plate embedded in a porous medium. Numerical Heat Transfer, Part A: Applications, 28(5), 635-645.

Khan, A., Ali Abro, K., Tassaddiq, A., \& Khan, I. (2017). Atangana-Baleanu and Caputo Fabrizio analysis of fractional derivatives for heat and mass transfer of second grade fluids over a vertical plate: a comparative study. Entropy, 19(8), 279-290.

Bejan, A., \& Khair, K.R. (1985). Heat and mass transfer by natural convection in a porous medium. International Journal of Heat and Mass Transfer, 28(5), 909-918.

Hossain, M.A., \& Munir, M.S. (2000). Mixed convection flow from a vertical flat plate with temperature dependent viscosity. International Journal of Thermal Sciences, 39(2), 173-183.

Hristov, J. (2017). Steady-state heat conduction in a medium with spatial non-singular fading memory: Derivation of Caputo-Fabrizio space-fractional derivative from Cattaneo concept with Jeffreys Kernel and analytical solutions. Thermal science, 21(2), 827-839. 
International Journal of Mathematical, Engineering and Management Sciences

Vol. 6, No. 2, 493-509, 2021

https://doi.org/10.33889/IJMEMS.2021.6.2.030

Javaherdeh, K., Nejad, M.M., \& Moslemi, M. (2015). Natural convection heat and mass transfer in MHD fluid flow past a moving vertical plate with variable surface temperature and concentration in a porous medium. Engineering Science and Technology, an International Journal,18(3), 423-431. https://doi.org/10.1016/j.jestch.2015.03.001.

Lai, F.C., \& Kulacki, F.A. (1990). The effects of variable viscosity on convective heat and mass transfer along a vertical surface in standard porous media. International. Journal of Heat and Mass Transfer, 33(5), 1028-1031.

Mirza, I.A., \& Vieru, D. (2017). Fundamental solutions to advection-diffusion equation with timefractional Caputo-Fabrizio derivative. Computers \& Mathematics with Applications, 73(1), 1-10. https://doi.org/10.1016/j.camwa.2016.09.026.

Mukhopadhyay, S., \& Layek, G.C. (2008). Effects of thermal radiation and variable fluid viscosity on free convective flow and heat transfer past a porous stretching surface. International Journal of Heat and Mass Transfer, 51(6), 2167-2178.

Shah, N.A., \& Khan, I. (2016). Heat transfer analysis in a second grade fluid over and oscillating vertical plate using fractional Caputo-Fabrizio derivatives. The European Physical Journal C, 76(7), 362. https://doi.org/10.1140/epjc/s10052-016-4209-3.

Sheikh, N.A., Ali, F., Saqib, M., Khan, I., Jan, S.A.A., Alshomrani, A.S., \& Alghamdi, M.S. (2017). Comparison and analysis of the Atangana-Baleanu and Caputo-Fabrizio fractional derivatives for generalized Casson fluid model with heat generation and chemical reaction. Results in Physics, 7, 789800 . 